\title{
Towards a Predictive Hybrid RANS/LES Framework
}

\author{
Sigfried Haering*, Todd A. Oliver ${ }^{\dagger}$, Robert D. Moser ${ }^{\ddagger}$ \\ The University of Texas at Austin, Austin, Texas, 78712
}

\begin{abstract}
Predictive simulation of many complex flows requires moving beyond Reynolds-averaged Navier-Stokes (RANS) based models to representations resolving at least some scales of turbulence in at least some regions of the flow. To resolve turbulence where necessary while avoiding the cost of performing large eddy simulation (LES) everywhere, a broad range of hybrid RANS/LES methods have been developed. While successful in some situations, existing methods exhibit a number of deficiencies which limit their predictive capability in many cases of interest, for instance in many flows involving smooth wall separation. These deficiencies result from inappropriate blending approaches and resulting inconsistencies between the resolved and modeled turbulence as well as errors inherited from the underlying RANS and LES models. This work details these problems and their effects in hybrid simulations, and develops a modeling paradigm aimed at overcoming these challenges. The new approach discards typical blending approaches in favor of a hybridization strategy in which the RANS and LES model components act through separate models formulated using the mean and fluctuating velocity, respectively. Further, a forcing approach in which fluctuating content is actively transferred from the modeled to the resolved scales is introduced. Finally, the model makes use of an anisotropic LES model that is intended to represent the effects of grid anisotropy. The model is demonstrated on fully-developed, incompressible channel flow and shown to be very promising.
\end{abstract}

\section{Introduction}

It is widely recognized that combining Reynolds-averaged Navier-Stokes (RANS) models with large eddy simulation (LES) in a hybrid modeling framework can, in principle, provide a computationally tractable, predictive tool for simulation of high Reynolds number flows in complex geometries. Since the 1990s, many hybrid simulation approaches, including Detached Eddy Simulation (DES) and variants [1]-5], partially-averaged Navier-Stokes [6, 7], partially-integrated transport models [8, 9], scale-adaptive simulation [10, 11], zonal RANS/LES approaches [12, 13], and others [14-16], have been developed. However, despite this research and the intuitive appeal of the ideas underlying hybrid models, because of a host of issues introduced by the blending of RANS and LES models as well as deficiencies inherited from the underlying models, a hybrid approach capable of reliably predicting a wide range of flows does not yet exist. The overall objective of this work is to develop such a predictive hybrid RANS/LES modeling framework. This paper makes two contributions towards this goal. First, it highlights a set of deficiencies common to many hybrid methods. Second, it introduces a general modeling framework in which these deficiencies can be addressed in a systematic fashion.

The deficiencies of existing methods come in three categories. The first is related to combining RANS and LES. Naturally, the hybridization of RANS and LES is at the heart of hybrid models. However, in most existing methods, the form of the transition between RANS and LES-like modeled stress is ad-hoc. The deficiencies of such blending lead to non-physical behavior of the blended state, thereby corrupting the hybrid simulation. As discussed in \$II.A. it appears a primary cause of these shortcomings is the blending of RANS and LES through a single stress model, which is invalid because the requirements and characteristics for the two models are incompatible. Instead, an alternative hybridization strategy is required.

Second, in complex hybrid simulations, fluid inevitably flows from RANS to LES regions and vice versa and between regions of varying LES resolution. These situations require that energy associated with turbulent fluctuations be exchanged between resolved and modeled scales. Most current methods affect this exchange passively, relying on natural instabilities and dissipation. However, as shown in \$[II.B, to make effective use of LES-like resolution while

\footnotetext{
*Postdoctoral Researcher, Institute for Computational Engineering and Sciences

${ }^{\dagger}$ Research Scientist, Institute for Computational Engineering and Sciences

${ }^{\ddagger}$ Professor, Institute for Computational Engineering and Sciences and Department of Mechanical Engineering
} 
avoiding the well-known modeled stress depletion (MSD) problem [17], it is necessary that the exchange between modeled and resolved turbulence be actively managed or forced.

Finally, in LES regions, hybrid models inherit the deficiencies of the subgrid stress (SGS) model formulations they are based upon. Typical LES models are built assuming isotropic unresolved turbulence and homogeneous, isotropic filtering/resolution. Most SGS models do not consider that these assumptions may be violated, leading to poor performance [17]. In hybrid applications, these shortcomings can be particularly acute because the grids usually have coarse LES resolution as well as anisotropic cells and inhomogeneous resolution. On such grids, the unresolved scales contribute a significant portion of the total turbulent stress and are still strongly anisotropic.

Overcoming these shortcomings necessitates a three-pronged approach. First, to address issues associated with blending, this work introduces a hybridization technique in which RANS and LES SGS models act through separate model formulations using the mean and fluctuating velocity fields, respectively. Second, the approach is equipped with a forcing mechanism that affects the exchange between resolved and modeled turbulence on time scales that allow the resolved turbulence to maintain local equilibrium, thereby avoiding the need to generate realistic synthetic turbulence. Third, the approach is able to make use of nearly any underlying RANS and LES models, allowing the inadequacies of typical models to be overcome as improved models are developed. For example, in this work, an anisotropic LES model that represents the effects of grid anisotropy is used. Taken together, these three developments are intended to resolve the problems noted previously.

The approach is demonstrated on fully-developed, incompressible, turbulent channel flow at $R e_{\tau}=5200$. The results show that the mean velocity is well predicted for a range of levels of resolved turbulence and that the turbulent kinetic energy is better predicted than in RANS. Thus, the new method appears promising, although further testing on a broader range of cases is of course necessary.

The remainder of the paper begins with a more in depth examination of the problems briefly discussed above in $\$$ Then, $\S[\mathrm{II}$ describes the approach developed here, and $\$[\mathrm{IV}$ shows preliminary results for channel flow. Finally, $\$ \mathrm{~V}$ provides conclusions.

\section{Motivation: Requirements for Predictive Hybrid RANS/LES}

As noted in \$ٓ there are three major issues in existing hybrid models. This section describes these issues in more detail, including examples of the errors they lead to in practical simulations.

\section{A. Inadequacy of Blending}

The RANS and filtered Navier-Stokes equations are formally similar, and common RANS and LES SGS models also take a similar form, where the effects of unresolved turbulence are represented through an eddy viscosity. These similarities lead to the alluring idea of blending the model stress such that the simulation transitions from pure RANS to full LES as a function of some measure of the available resolution. However, closure models built for RANS and LES have conflicting characteristics that make it difficult, if not impossible, to successfully blend them through a single eddy-viscosity-based stress model. There are two primary issues. First, typical RANS transport equations are not designed for use with fluctuating state. Second, typical LES models are not designed to simultaneously predict the subgrid contribution to the Reynolds stress and the dissipation.

\section{RANS model behavior with fluctuating state}

One problem is that transport equation models built for use in RANS may severely misbehave when applied to LES fluctuations. For example, consider the destruction term in the standard model for the evolution of the turbulent dissipation rate $\varepsilon: \mathcal{D}_{\varepsilon}=C_{\varepsilon 2} \varepsilon^{2} / k$. As an approximation of the destruction term in the exact $\varepsilon$ equation, this form has little physical justification. Nonetheless, for RANS, the model can be collectively calibrated to behave reasonably well. However, in an LES, the fluctuations have a substantial impact on the term's mean value. To illustrate the impact of the fluctuations, one can expand $1 / k$ in a Taylor series about $1 /\langle k\rangle$. Retaining the linear and quadratic terms in $k^{\prime}$ leads to the following approximation:

$$
\left\langle\frac{\varepsilon^{2}}{k}\right\rangle \approx \frac{\langle\varepsilon\rangle^{2}}{\langle k\rangle}\left(1+\frac{\left\langle\varepsilon^{\prime 2}\right\rangle}{\langle\varepsilon\rangle^{2}}+\frac{\left\langle k^{\prime 2}\right\rangle}{\langle k\rangle^{2}}-\frac{\left\langle\varepsilon^{\prime} k^{\prime}\right\rangle}{\langle\varepsilon\rangle\langle k\rangle}-\frac{\left\langle\varepsilon^{\prime 2} k^{\prime}\right\rangle}{\langle\varepsilon\rangle^{2}\langle k\rangle}+\frac{\left\langle\varepsilon^{\prime} k^{\prime 2}\right\rangle}{\langle\varepsilon\rangle\langle k\rangle^{2}}+\frac{\left\langle\varepsilon^{\prime 2} k^{\prime 2}\right\rangle}{\langle\varepsilon\rangle^{2}\langle k\rangle^{2}}\right) .
$$


While it is not obvious what correlations may arise from all the terms, the variances in $k^{\prime}$ and $\varepsilon^{\prime}$ are clearly positive definite with fluctuations present. Due to non-zero variances and covariances when $k$ and $\varepsilon$ are fluctuating, $\left\langle\mathcal{D}_{\varepsilon}\right\rangle$ is not simply $C_{\varepsilon 2}\langle\varepsilon\rangle^{2} /\langle k\rangle$ as it is in RANS. Instead, the behavior of the entire system of equations is altered depending on how much turbulence is resolved.

Results from hybrid simulations of channel flow at $R e \approx 5200$ (see Appendix, "Model C" for model details) confirm the dramatic effect of fluctuations on the mean source terms in the $k$ and $\varepsilon$ equations. Figure 1 shows the difference between the mean of the fluctuating production of $k$ and $\varepsilon$, and destruction of $\varepsilon$ and the values of these

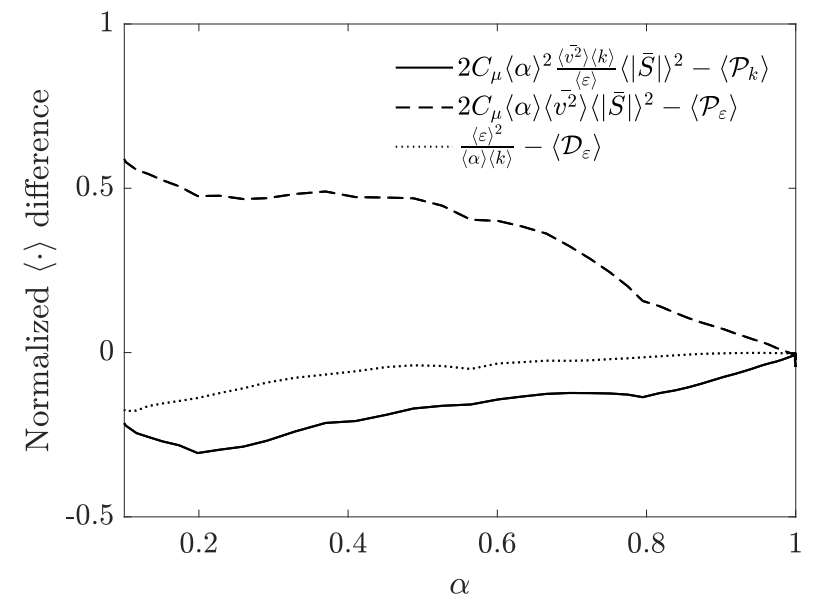

Fig. 1 Differences in source terms for $k$ and $\varepsilon$ as calculated from the means of the input quantities and the mean of the full fluctuating term. The production of $k$ is normalized by $\left\langle\mathcal{P}_{k}\right\rangle$ while both production and destruction of $\varepsilon$ are normalized $\left\langle\mathcal{D}_{\varepsilon}\right\rangle$.

quantities evaluated at the mean. As the fraction of turbulent kinetic energy that is modeled $(\alpha)$ goes down, $\mathcal{P}_{k}$ and $\mathcal{D}_{\varepsilon}$ are enhanced by the fluctuations, and $\mathcal{P}_{\varepsilon}$ is diminished. In a channel flow, the result is that in the outer region, where $\alpha$ is small, $k$ is enhanced and $\varepsilon$ reduced relative to RANS. This modifies the subgrid stress, which then distorts the mean velocity, as shown in Figure 2

Transport-based $k$-SGS models have been used for some time [18], without this difficulty. However, in those cases, the model length scale is taken as the grid scale, which results in production and dissipation of kinetic energy scaling like $k^{1 / 2}$ and $k^{3 / 2}$, respectively. The stronger dependence of dissipation on $k$ effectively damps the $k$ fluctuations, minimizing the effects of this problem. But in a hybrid context, this would cause new problems, such as resolved and modeled turbulence that is out of equilibrium due to MSD, and the lack of an intrinsic model length scale to use in hybridization. For instance, when the SGS lengthscale is between the integral and grid lengthscales, as will occur in hybrid simulations, the production scaling with $k^{1 / 2}$ is entirely incorrect. To fully address this problem, the RANS transport equations should be used to represent mean quantities as a function of only mean quantities, as designed.

\section{Stress/dissipation inconsistency in subgrid-viscosity-based LES}

In addition to problems that stem from using RANS-like transport equations with resolved fluctuations, there are also problems that arise when typical subgrid viscosity-based models are used in LES regions where a significant fraction of the mean turbulent stress is not resolved.

The issue here is an inherent inconsistency between the requirements of predicting the subgrid contribution to the Reynolds stress and the dissipation (or, more correctly, the transfer of kinetic energy from resolved to unresolved scales) when using a subgrid-viscosity-based LES model, as discussed by Jiménez and Moser [19]. In particular, because of the model structure, the subgrid stress and strain are fully correlated, which is physically incorrect. Analysis suggests a correlation of $20-30 \%$ for isotropic turbulence. This incorrect correlation would lead to incorrect dissipation except that the models have calibration coefficients, e.g., $C_{S}$ for Smagorinsky, whose value can be selected to give the correct dissipation. However, this calibration then causes the subgrid Reynolds stresses to be incorrect-i.e., it is not possible to simultaneously match both dissipation and subgrid Reynolds stresses in eddy-viscosity-based models. The outcome is that the subgrid contributions to the Reynolds stress predicted by typical LES models are often extremely wrong. 


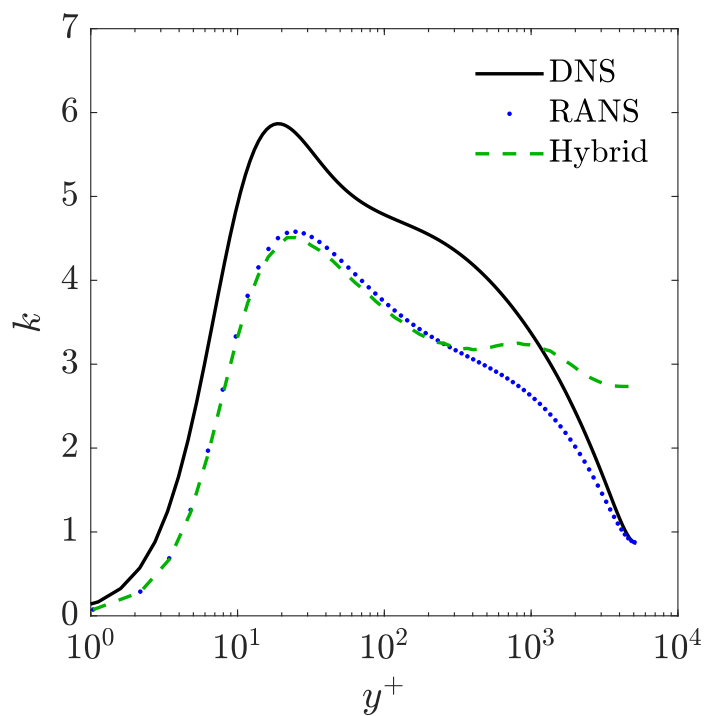

(a) TKE

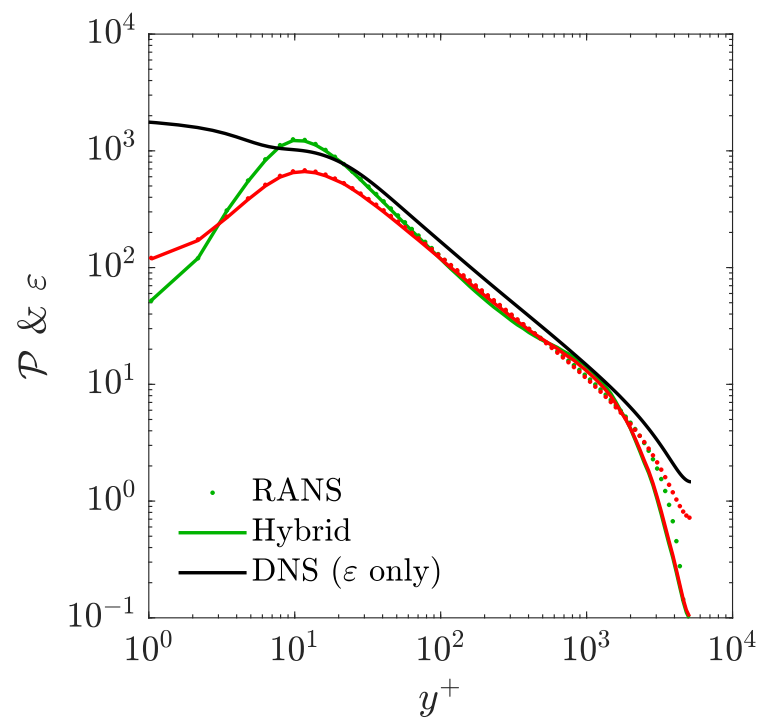

(b) $k$ source terms

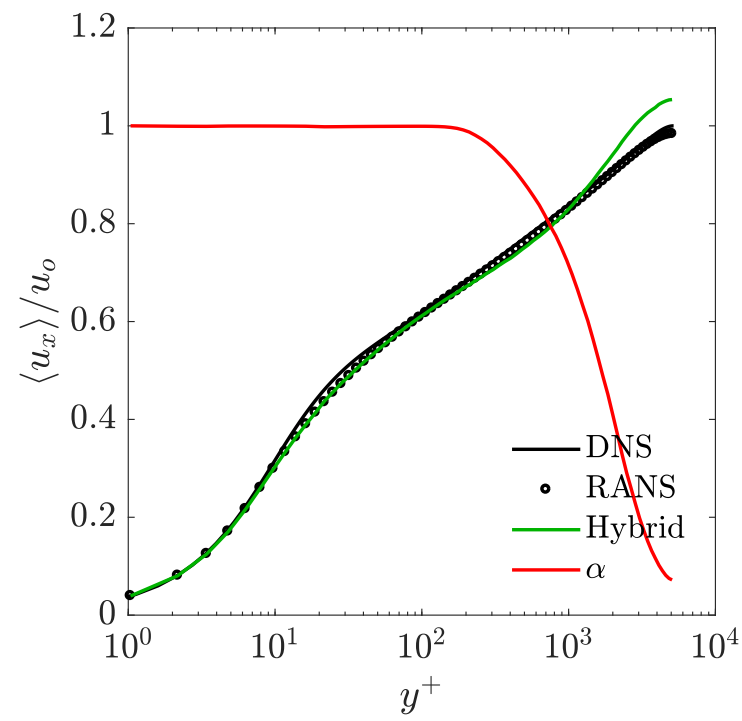

(c) Mean velocity

Fig. 2 Turbulent kinetic energy (a), production and destruction of TKE (b), and mean velocity (c) for turbulent channel flow. For (b), the black solid line is the DNS dissipation and (-) is used for production while $(-)$ is used for dissipation. 
For instance, Jiménez and Moser [19] show that dynamic Smagorinsky predicts a subgrid Reynolds shear stress that is roughly a factor of five lower than shear stresses obtained from filtered DNS for channel flow.

In pure LES, the impact of this problem can be minimized by using sufficient resolution so that the subgrid shear stresses are negligible. In this case, only the dissipation is important. Alternatively, in pure RANS these issues do not arise because the model is only required to predict the Reynolds stress and not the dissipation. Unfortunately, the problem is unavoidable in hybrid methods. In regions with a modest level of resolved turbulence, but not enough to dominate the total stress, the model must accurately represent both the energy transfer from resolved to modeled scales and the subgrid contribution to the total stress. These observations suggest that blending through a single eddy-viscosity-based stress model does not provide a sufficiently rich modeling structure to accurately model both roles of the subgrid term.

\section{B. Active Turbulence Management}

While a new paradigm for combining RANS and LES is necessary, this alone is not sufficient for a predictive hybrid model. Recent hybrid simulations of the NASA wall-mounted hump (WMH) test case [20] indicate that a mechanism for active exchange of energy between the resolved and modeled scales is also necessary. This conclusion is based on results from two variants of a hybrid model (see Appendix for model details) developed by the authors. Both of these models accomplish blending by lowering the model stress on the time scale associated with the modeled turbulence in regions where turbulence can be supported. The idea being that by lowering the stress gradually the natural instabilities of the flow would produce resolved fluctuations to avoid stress depletion.

Results are shown in Figure 3 and 4 In the first version of the model, labeled Model A, some resolved fluctuations are obtained in the outer portion of the boundary layer on the hump upstream of the separation point. These fluctuations are essential to initiate the correct break-up of the separated shear layer necessary to accurately predict the reattachment point. The Model A prediction of the reattachment point agrees well with experiments. However, Model A leads to a poor prediction of the skin friction coefficient upstream of and on the hump (Fig. 44), indicating that the fluctuations are not sufficient to offset the drop in wall normal momentum transfer due to reduced modeled stress.

This stress depletion problem can be avoided by enhancing the modeled wall-normal transport as is done via an anisotropic eddy viscosity in Model B leading to accurate skin friction upstream of the separation. However, this modification also suppresses fluctuations upstream of the separation, keeping the solution more RANS-like in that region. The resulting lack of fluctuations flowing into the separated shear layer leads to delayed roll-up and an inaccurate prediction of the reattachment point. Qualitatively, this behavior is similar to DDES, which is also RANS-like near the separation point and reattaches too far downstream.

The takeaway from these results is that to correctly predict both skin friction and shear layer roll-up simultaneously, their must be resolved turbulence in the outer portion of the boundary layer and the model must ensure the resolved and modeled turbulence are in agreement; the only way to achieve this everywhere in a hybrid simulation is by actively creating resolved turbulence where the grid can support fluctuations, particularly in low shear regions where the instabilities in the flow are insufficient to rapidly self-generate turbulence.

\section{Effects of Anisotropy in LES}

It is well understood that standard SGS models formulated using assumptions of isotropic subgrid turbulence and isotropic resolution break down when these conditions are violated. In previous work [21], a number of SGS models were evaluated in forced, homogeneous, isotropic turbulence on anisotropic grids. The results show that, for mildly anisotropic cells, the Smagorinksy model leads to under dissipation in coarse directions, incorrect energy distribution across fine scales manifesting in an incorrect inertial range slope, and over dissipation in the fine directions. Being under dissipative in coarse directions and at lower wavenumbers in the fine directions is especially problematic as it leads to spurious oscillations and can corrupt an entire simulation. These problems are particularly acute in hybrid simulations of complex geometries because highly anisotropic, coarse resolution is typically present.

Elimination of excess energy at lower wavenumbers is the most critical component in selecting the form of the SGS model anisotropy. More recent subgrid models, such as the anisotropic minimum dissipation (AMD) model [22] and the M43 model [21] have, to some extent, overcome the problems observed with Smagorinsky for isotropic turbulence and anisotropic resolution. However, in traditional hybrid schemes, it is not clear how to transition between the anisotropic behavior of these models, which are related to energy transfer and not stress, with RANS. This further motivates the development of a new hybridization approach that can naturally make use of such models. 

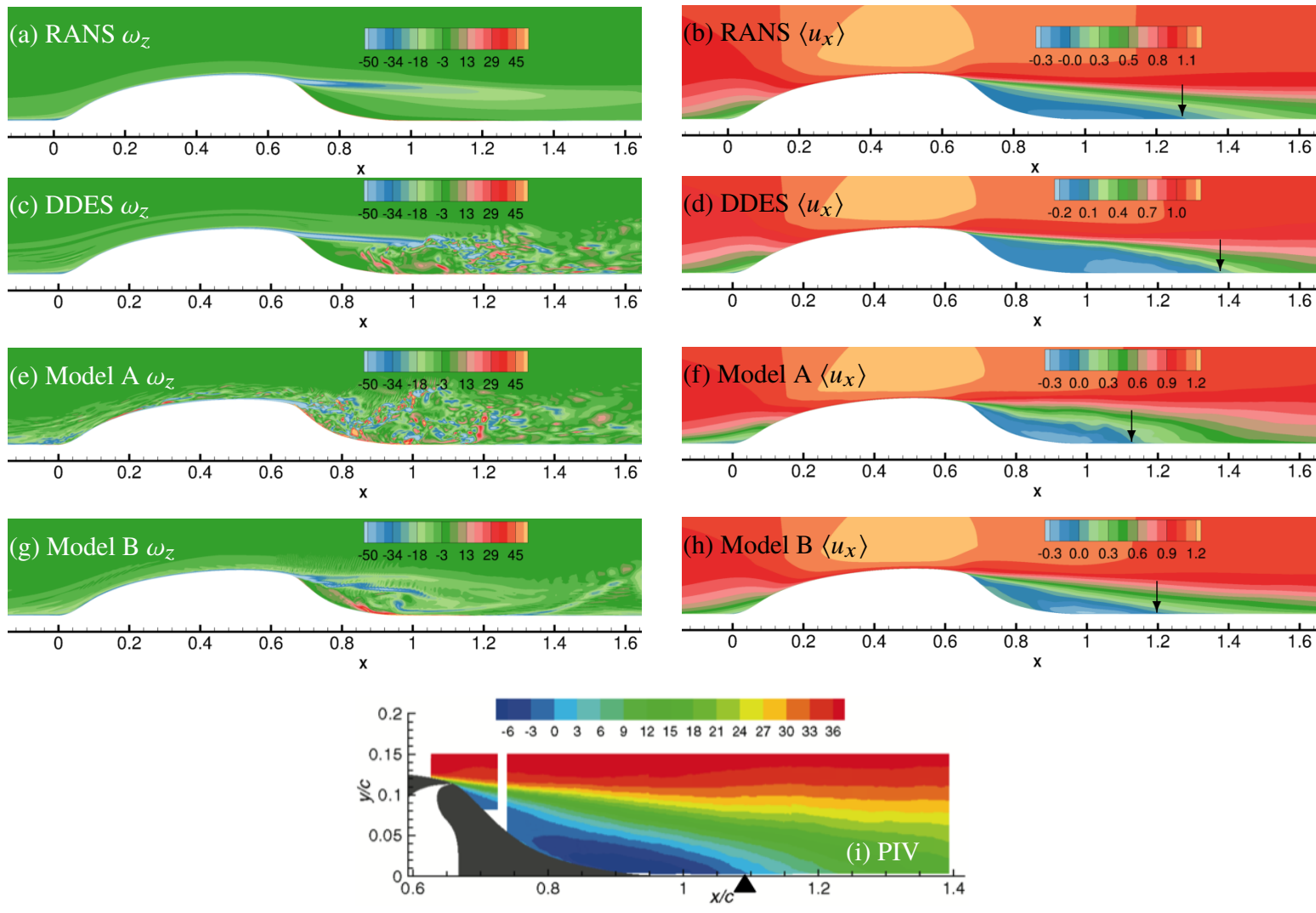

Fig. 3 Results for two hybrid model variants used to simulate the NASA wall-mounted hump, compared to $\overline{v^{2}}-f$ RANS, DDES, and experiment: Instantaneous spanwise vorticity (a,c,e,g) and mean streamwise velocity (b,d,f,h,i). The black arrows indicate reattachment location.

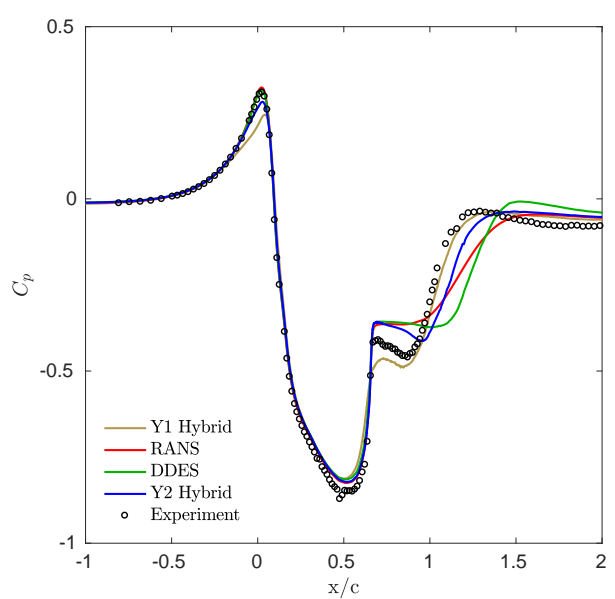

(a) $C_{p}$

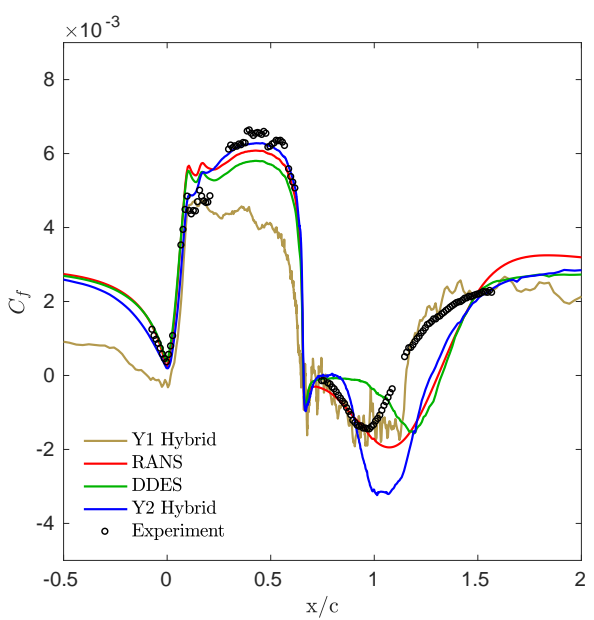

(b) $C_{f}$

Fig. 4 Model A (Y1) and Model B (Y2) NASA wall-mounted hump results, compared to $\overline{v^{2}}-f$ RANS and DDES: pressure coefficient (left) and skin friction coefficient (right). 


\section{Developments Enabling Predictive Hybrid RANS/LES}

This section describes a modeling approach designed to overcome the deficiencies described in $\$$ II In particular, a novel hybridization paradigm based on separating the RANS and standard subgrid contributions to the model term is introduced in \$II.A. The model requires an active energy transfer from modeled to resolved scales. A preliminary approach for this forcing is outlined in \$III.B Finally, an LES model designed for use with anisotropic grids is described in $\$$ III.C.

\section{A. Hybridization via Model-Splitting}

Motivated by the drawbacks of blending RANS and LES through a single, eddy-viscosity-based stress model, as described in \$II.A the hybridization strategy introduced here is based upon splitting the total model stress into two separate portions: one primarily responsible for the mean subgrid stress and one mainly representing energy transfer to the subgrid. Thus, in this "model-split" (MS) hybridization, the resolved momentum equation takes the following form:

$$
D_{t} \bar{u}_{i}=-\frac{1}{\rho} \partial_{i} \bar{p}+v \partial_{j} \partial_{j} \bar{u}_{i}+\partial_{j}\left(\tau_{i j}^{S G R S}+\tau_{i j}^{S G E T}\right)+F_{i},
$$

where $\bar{u}_{i}$ is the resolved velocity, $\bar{p}$ is the resolved pressure, $\tau_{i j}^{S G R S}$ is intended to model the mean subgrid stress, $\tau_{i j}^{S G E T}$ is used to represent the energy transfer from the resolved to modeled scales, and $F_{i}$ is used to induce the transfer of energy from the modeled to resolved scales.

To further develop this approach, the total velocity field is decomposed as $u_{i}=\langle u\rangle_{i}+u_{i}^{\prime}=\langle u\rangle_{i}+u_{i}^{>}+u_{i}^{<}$, where $\langle u\rangle_{i}$ is the Reynolds averaged velocity, and turbulent velocity fluctuations $\left(u_{i}^{\prime}\right)$ are divided into resolved $\left(u_{i}^{>}\right)$and discarded $\left(u_{i}^{<}\right)$components; i.e., the total resolved velocity field is $\bar{u}_{i}=\langle u\rangle_{i}+u_{i}^{>}$. The resolved momentum flux is $\left(u_{i}^{>}\langle u\rangle_{j}+\langle u\rangle_{i} u_{j}^{>}+u_{i}^{>} u_{j}^{>}\right)$so that the average turbulent stress to be modeled is $\tau_{i j}^{S G R S} \approx\left\langle u_{i}^{\prime} u_{j}^{\prime}\right\rangle-\left\langle u_{i}^{>} u_{j}^{>}\right\rangle$. Assuming that both stress tensors scale with their trace, then $\tau_{i j}^{S G R S} \approx \alpha\left\langle u_{i}^{\prime} u_{j}^{\prime}\right\rangle$ where $\alpha$ is the ratio of modeled to total TKE: $\alpha=k_{s g s} / k_{\text {tot }}=\left(1-k_{\text {res }} / k_{\text {tot }}\right)$, where $k_{\text {res }}=\left\langle u_{i}^{>} u_{i}^{>}\right\rangle / 2$. Hence, a simple model for the average subgrid stress would be $\tau_{i j}^{S G R S}=\alpha \tau_{i j}^{R A N S}$, where $\tau_{i j}^{R A N S}$ denotes a RANS closure evaluated, as in standard RANS, using only mean quantities.

This scaling represents a dramatic simplification of the true behavior of the subgrid stress as a function of the Reynolds stress and resolved turbulence. The true scaling likely depends on proximity of the subgrid lengthscale to the integral and Kolmogorov lengthscale. Indeed, the similarity analysis of Lumley [23], as discussed in [19], suggests that, for even low mean shear, the anisotropic portion of the modeled shear stress decays like $\alpha^{2}$, i.e., faster than the subgrid $k$. Thus, the model is known to be wrong in some sense. However, when coupled with existing RANS models, scaling the Reynolds stress anisotropy by $\alpha^{2}$ was found to perform worse than $\alpha$. This behavior is attributed to the fact that the scalar quantities, e.g., $k$ and $\epsilon$, in RANS models are only loosely representative of the corresponding true quantities. RANS models function by a series of error-canceling and coefficient tuning to arrive at nearly the correct mean stress provided the mean gradients; i.e., they get the "correct" eddy viscosity and not the correct individual scalar contributions to the eddy viscosity. Thus, it is possible that the theoretically correct scaling for any given flow and level of resolved turbulence will not perform well in a hybrid simulation built upon existing inadequate models. Because of this, the $\alpha$ scaling is used here, but improving, or removing the necessity for, this scaling will be the subject of future works.

Regarding energy transfer, since $\tau_{i j}^{S G R S}$ is not fluctuating, it transfers kinetic energy from the mean to the unresolved scales, but not from the resolved fluctuations to the unresolved scales. Instead, the latter transfer is modeled through $\tau_{i j}^{S G E T}$. Traditional LES SGS models primarily function to model this transfer with the majority of the turbulence stress being resolved. Accordingly, one may use typical LES SGS model forms for $\tau_{i j}^{S G E T}$. In theory, any standard SGS model may be applicable however, we have performed MS simulation with the model in \$III.Chere. Energy transfer to the subgrid is largely a result of the interactions of scales of motion near the cutoff [24] and only indirectly depends on mean gradients. Thus, only the fluctuating part of the resolved velocity gradient is used to evaluate $\tau_{i j}^{S G E T}$. For typical model forms, $\tau_{i j}^{S G E T}$ may contribute to the mean stress due to non-vanishing correlations between the model viscosity and fluctuating velocity gradient tensor. However, for some models, such as that described in \$III.C. $\left\langle\tau_{i j}^{S G E T}\right\rangle=0$, perfectly delineating the roles of the two terms. If the $\tau_{i j}^{S G E T}$ model is selected so that the SGET eddy viscosity is uncorrelated with the fluctuating gradient, the MS approach can be considered as a method of segregating the mean and fluctuating portions of the modeled stress and isolating their eddy-viscosity dependencies. That is, the total model is decomposed into the mean deviatoric stress $\left(\tau_{i j}^{S G R S}=\left\langle\tau-\frac{2}{3} k_{s g s} I\right\rangle\right)$ and the fluctuating portion 
$\left(\tau_{i j}^{S G E T}=\tau-\tau_{i j}^{S G R S}\right)$

Due to the necessity of knowing $k_{t o t}$, any two-equation RANS model should be functional in the MS framework. In a $k$-based model, the model equations can be used unchanged except for the production term, which is modified as follows:

$$
\mathcal{P}_{k}=\left\langle S_{i j}\right\rangle\left(\tau_{i j}^{S G R S}+\left\langle\tau_{i j}^{S G E T}\right\rangle-\left\langle u_{i}^{>} u_{j}^{>}\right\rangle\right),
$$

where the first term is already an expected quantity by construction and the second term will generally be small, or zero, depending on the selection of the SGET model (see \$III.C). Naturally, all time and length scales used in the RANS model must remain the mean values, and not subgrid. Other modifications to the RANS model transport equations, e.g., to use information from the fluctuating state in the transport closures, can be imagined, but are not used here. To use a one equation model, such as SA [25], further modeling to obtain $k_{t o t}$ is necessary. For example, one can approximate $k_{t o t}$ from the eddy viscosity and a timescale extracted directly from the mean velocity gradient tensor. Such an approach is likely to work in flows with one primary inhomogeneous direction, such as channel flow or 2-D boundary layers. How to extend the approach to more general flows is left for future work.

The model-split hybridization approach has numerous advantages over traditional blending methods. First, it uses the RANS model as designed. The turbulence model state variables represent mean features of the turbulence, and the governing PDEs depend only on mean quantities. Thus, pathological behaviors of the RANS transport models due to fluctuating state variables are avoided. Second, the RANS eddy viscosity appearing in $\tau_{i j}^{R A N S}$ makes no contribution to the dissipation of resolved fluctuations, allowing turbulence at all resolvable scales to form naturally without being overly dissipated. Third, nearly any combination of base mean and fluctuating models can be used. Because of this flexibility, advanced models are easily incorporated, which is necessary to treat the effects of anisotropy, as discussed in $\$$ III.C.

While there are many advantages, the model-split form introduces new challenges as well. For one, in the above development, we have tacitly assumed knowledge of the expected value for all required quantities, yet this information is not automatically available in hybrid simulations. Thus, a method for obtaining the mean quantities is required. For stationary flows, a causal time average with an exponentially decaying kernel should be sufficient, and this approach is used here. In this case, the mean of a quantity $\phi$ evolves according to

$$
d_{t}\langle\phi\rangle=\frac{1}{T_{\text {ave }}}(\bar{\phi}-\langle\phi\rangle),
$$

where the time constant is the large-scale turbulent time scale, or $T_{\text {ave }}=k_{\text {tot }} / \varepsilon$. In this way, the majority of the turbulent fluctuations, $\phi^{>}$, will be removed from $\langle\phi\rangle$.

\section{B. Active Energy Transfer}

Based on the work described in $\$ \Pi$ II.B it has become clear that active exchange of energy between resolved and unresolved scales (forcing) is a requirement for a robust hybrid modeling framework. In particular, in regions that are able to resolve more turbulence, velocity fluctuations should be added at the length scale of the smallest resolved turbulence. On the other hand, in under-resolved regions, $\tau_{i j}^{S G E T}$ will naturally move energy from the resolved to the

modeled scales. In general, due to grid inhomogeneity, $\tau_{i j}^{S G E T}$ alone may be insufficient to affect this transfer fast enough. In this initial work, the forcing scheme is designed only to inject fluctuations into RANS and near-RANS regions that can support more resolved turbulence. Modification to $\tau_{i j}^{S G E T}$ or $F_{i}$ to rapidly remove resolved energy in response to rapidly coarsening resolutions will be a subject of future work. Active forcing of the resolved field requires the ability to: 1) identify regions where more turbulence can be resolved, 2) determine the rate at which resolved kinetic energy should be added, and 3) specify the structure of the velocity fluctuation to be created.

Identifying regions where forcing is possible and/or needed requires evaluating the grid's ability to resolve more or less turbulence, which is a common requirement of hybrid models. Typical measures compare scalar measures of grid spacing (e.g., cell diagonal or volume cube-root) to a scalar turbulent length scale. For anisotropic grids and/or turbulence such measures are clearly incomplete indicators and generally insufficient. Instead, the evaluation should be based on the locally least-resolved orientation to ensure adequate resolution in all directions. Naturally, this measure must depend on both the grid and the flow state. Here, an anisotropic resolution adequacy metric is 
constructed from the ratio of the grid spacing to the length scale of the turbulence produced by the model:

$$
r_{\mathcal{M}}=C_{r} \max _{\forall e}\left(\mathcal{L}_{\mathcal{P}}^{-1} \cdot \mathcal{M}\right) \approx\left(\frac{3}{2 \overline{v^{2}}}\right)^{3 / 2} \max \left(\mathcal{P}_{i k}^{s g s} \mathcal{M}_{k j}\right)
$$

where $C_{r}$ is a constant (currently set to 1 ) related to how many grid sizes are required to resolved a turbulent structure of size $\mathcal{L}_{\mathcal{P}}, \overline{v^{2}}$ is the variance of the wall-normal velocity fluctuations, $\mathcal{P}_{i j}^{s g s}$ is the subgrid production tensor (not the the RANS production),

$$
\mathcal{P}_{i j}^{s g s}=\frac{1}{2}\left(\tau_{i k} \partial_{j} \bar{u}_{k}+\tau_{j k} \partial_{i} \bar{u}_{k}\right),
$$

where the full subgrid stress, including the mean isotropic portion, is considered

$$
\tau_{i j}=\tau_{i j}^{S G R S}+\tau_{i j}^{S G E T}+\frac{2}{3} \alpha k_{t o t} \delta_{i j}
$$

and $\mathcal{M}_{i j}$ is a tensor characterizing the anisotropic resolution (square-root of symmetric part of metric tensor defining mapping from a unit cube to the physical cell [26]). The resolution tensor is composed of eigenvalues describing the cell orientation and eigenvalues being common grid dimensions $\left(\delta_{x}, \delta_{y}, \delta_{z}\right)$ with its invariants being common ambiguous grid measures, i.e. $3 \delta_{\text {ave }}=\mathcal{M}_{k k}, \delta_{\text {diag }}^{2}=\left(\mathcal{M}_{i j} \mathcal{M}_{i j}\right), \delta_{v o l}^{3}=\operatorname{det}(\mathcal{M})$. The isotropic portion of $\tau$ is typically ignored in favor of a modified pressure gradient as it does not actually produce any turbulent kinetic energy. However, in this context it is necessary for evaluating the directional production of subgrid turbulence. The quantity $\overline{v^{2}}$ is naturally available in Durbin's $\overline{v^{2}}-f$ model [27], but can also be approximated using any other two-equation turbulence model as $\overline{v^{2}} \approx 5 v_{t} / T$, where $T$ is the model turbulence time scale (for instance $T \approx 1 /\left(\beta^{*} \omega\right)$ for the SST model). Thus, its presence in (3) does not restrict the method to the $\overline{v^{2}}-f$ model but is merely a vehicle for incorporating near-wall effects.

This resolution adequacy measure is the ratio of the resolution length scale to the length scale of turbulence produced by the model. Thus, when $\left\langle r_{\mathcal{M}}\right\rangle>1$, the simulation is under-resolved, and vice versa when $\left\langle r_{\mathcal{M}}\right\rangle<1$. For isotropic grids with isotropic turbulence in equilibrium, $\left\langle r_{\mathcal{M}}\right\rangle$ reduces to the standard DES length scale comparison. Thus, it may be viewed as a tensor generalization of this approach. In regions where $\left\langle r_{\mathcal{M}}\right\rangle<1$, the goal of forcing is to introduce some resolved fluctuation at a gradual rate allowing the forced resolved structures to evolve into actual turbulence thereby not corrupting the mean. These goals and dimensional consistenty suggest that the magnitude of the forcing acceleration should be based on the largest of the unresolved fluctuations and the subgrid timescale. Making use of near-wall anisotropy, the target forcing is defined as

$$
\left|F_{t a r}\right|=C_{F} \frac{\sqrt{\alpha \overline{v^{2}}}}{\alpha T}
$$

However, uniform forcing at $\left|F_{t a r}\right|$, over some region where $\left\langle r_{\mathcal{M}}\right\rangle<1$, is not desirable, as it would necessarily result in forcing the mean. Instead, in this work, we prescribe the spatial structure of the acceleration field and allow for $\left|F_{\text {tar }}\right|$ to only be attained where that artificial structure and the resolved turbulence allow. To this end, an auxiliary field is defined based on the structure of a Taylor-Green (TG) vortex with variable length scale. This TG field is as follows:

$$
\begin{aligned}
& h_{1}(x, t)=A \cos \left(a_{1} x_{1}^{p}\right) \sin \left(a_{2} x_{2}^{p}\right) \sin \left(a_{3} x_{3}^{p}\right), \\
& h_{2}(x, t)=B \sin \left(a_{1} x_{1}^{p}\right) \cos \left(a_{2} x_{2}^{p}\right) \sin \left(a_{3} x_{3}^{p}\right), \\
& h_{3}(x, t)=C \sin \left(a_{1} x_{1}^{p}\right) \sin \left(a_{2} x_{2}^{p}\right) \cos \left(a_{3} x_{3}^{p}\right),
\end{aligned}
$$

where the magnitudes are somewhat arbitrary but are selected such that $h_{i} h_{i} \leq 1$ with $A=1, B=-1 / 3$, and $C=-2 / 3$. The vortex sizes are set to mimic to local length scale at the cutoff, $\ell=\min \left(N_{L} L_{s g s}, \delta_{\text {wall }}\right)$, where $L_{s g s}=\left(\alpha k_{t o t}\right)^{3 / 2} / \varepsilon, \delta_{\text {wall }}$ is the distance to the wall, and $N_{L}=1$. This is accomplished by letting

$$
a_{i}=\pi /\left(D_{i} / \operatorname{nint}\left(D_{i} / \min \left(\max \left(\ell, 2 \ell_{i}^{\mathcal{M}}\right), D_{i}\right)\right)\right)
$$

where $\ell_{i}^{\mathcal{M}}=\left(r_{i} r_{i}\right)^{1 / 2}$ with $r_{i}=\mathcal{M}_{i j} e_{j}$ for each global coordinate direction $e_{i}$, and $D_{i}$ is the domain size in periodic directions. The consideration for periodic domains can be set to a very high number or simply removed $\left(a_{i}=\pi / \max \left(\ell, 2 \ell_{i}^{\mathcal{M}}\right)\right)$ in non-periodic directions. The TG vortex coordinates, $x_{i}^{p}$, are selected to translate with the 
mean flow to provide some degree of temporal correlation in addition to the desired length scale

$$
x_{i}^{p}(x, t)=x_{i}+\left\langle u_{i}\right\rangle t .
$$

With the target acceleration magnitude (6) and structure (9) in hand, additional modifications are necessary to respect RANS and DNS limits while also acknowledging the combination of $h_{i}$ and existing turbulent fluctuations would generally result in no net resolved production. The latter is addressed by testing the resolved production due to $h_{i}$ as

$$
\mathcal{P}_{F}^{\text {test }}=\left(\left|F_{\text {tar }}\right| \Delta t\right) h_{i} u_{i}^{>},
$$

which will be used to clip (9). The RANS limit is readily apparent when $\alpha=1$ while identification of near-DNS conditions requires an approximation of the local Kolmogorov length scale. With the information provided by the RANS model, this limit is found as $\alpha_{k o l}=C_{v}(v \varepsilon)^{1 / 2} / k_{t o t}$ with $C_{v}=1$. The effects of these three limiters are incorporated in a single scaling coefficient as

$$
\eta=-\left|F_{\text {tar }}\right|\left\{\begin{array}{l}
\min \left(S_{r}-D_{r}-F_{r}, 0\right) \text { if } \mathcal{P}_{F}^{\text {test }} \geq 0 \\
0 \text { otherwise }
\end{array}\right.
$$

where

$$
\begin{gathered}
D_{r}=\left(1+\alpha_{k o l}-\alpha\right) \min \left(S_{r}, 0\right), \\
F_{r}=\alpha \max \left(S_{r}, 0\right),
\end{gathered}
$$

and

$$
S_{r}=\tanh \left(\log \left(\left\langle r_{\mathcal{M}}\right\rangle\right)\right)
$$

is a function that gradually turns on forcing based on $\left\langle r_{\mathcal{M}}\right\rangle$. The forcing vector is simply

$$
F_{i}=\eta h_{i} .
$$

The above outlined approach is rudimentary. While it is clear that some forcing is required, what form it should take is not. The problem of introducing progressively more refined turbulent structures in an LES requires these structures to be synthetically generated. One way to view $F_{i}$ is as a model for the commutation error of the filtering operation and the substantial derivative, $\overline{D u_{i}} / D t-D \bar{u}_{i} / D t$, in the momentum equation induced by time- and space-dependent filter width. From this perspective, the only way to avoid "making up" fluctuations would be to have the full DNS solution at every step of an LES which would, of course, obviate the LES.

Existing forcing methods have typically addressed this issue by generating fluctuations only at a prescribed LES inlet [28, 29]. In addition to requiring specification of the distinct LES region and entire spectrum of locally resolved synthetic turbulent scales and intensities, the LES inlet location must be sufficiently upstream of the actual feature of interest so that the artificial inflow condition can "heal" to a realistic state. Body forcing may be thought of as a method to introduce synthetic turbulence at a "healing" rate so that, through the entire forced region, the flow maintains a realistic state. In this way, none of the simulated domain and associated computational cost is sacrificed allowing the entire simulated solution to yield useful information. The method proposed here is somewhat similar to the body forcing in the SAS-F model of Menter et.al. [30]. However, explicit time step dependance has been removed, and the structure is based on the variable length scale TG field and subgrid velocity scale rather than relying on generation of a wide spectrum of fluctuations using the Random Flow Generator method [31, 32]. By restricting the forcing structure to be only at the scale of the smallest locally resolved turbulence, existing larger, and presumably realistic, structures are not corrupted.

Nonetheless, the particular method outlined here has a number of potential drawbacks. First and foremost, the resulting $F_{i}$ is not divergence free. This is a result of TG fields only being divergence-free when constructed using a uniform overall scaling and vortex length scale. In an incompressible solver, the dilatational portion is projected out, which alters the structure of the effective forcing. The result is that the forcing can dissipate resolved turbulence at some points in space and time. However, on average, the approach does result in net production of resolved fluctuations. In a compressible solver, the method may result in spurious acoustic sources. Second, it should be possible to force more strongly, i.e., increase the coefficient $C_{F}$ (currently set to 8), by making the forcing more representative of some acceleration which would produce a real state of turbulence with length scales between $L_{s g s}^{n}$ and $L_{s g s}^{n+1}$ over some $\Delta t$ 
for the particular $\bar{u}_{i}$. That is, if the forcing were more representative of the commutation error $\overline{D u_{i}} / D t-D \bar{u}_{i} / D t$, generation of realistic resolved content could be affected more efficiently. There is no reason to expect the augmented TG field to be representative of these effects. By improving upon this structure, shorter lengths of LES resolution upstream to areas of interest, such as flow separation, will be necessary.

\section{Anisotropy Treatments}

In practical flows of engineering interest, the combination of high Reynolds number with complex geometries and limited computational resources often dictates discretization using relatively high aspect ratio (AR) cells of varying shape and rapidly varying size in space. In such cases, the assumptions of isotropic unresolved turbulence in equilibrium with the large scales and isotropic LES filtering will often be violated. Accordingly, issues of both turbulence and resolution anisotropy must be addressed.

In previous work, we applied a resolution-scale anisotropy representation to the entire LES model term by introducing a direction-dependent eddy viscosity [33]:

$$
\tau_{i j}=v_{j m} \partial_{m} \bar{u}_{i}+v_{i n} \partial_{n} \bar{u}_{j}
$$

with multiple tensor eddy viscosity models having been constructed and evaluated [34]. In light of the model-splitting method (\$II.A), the generalization of this approach requires two eddy viscosity tensors. Consistent with the purpose of each component of the subgrid model, model anisotropy can be divided into two main categories: turbulent stress anisotropy and dissipation anisotropy. Stress anisotropy describes how momentum is transferred differently between each velocity component in the flow. Dissipation anisotropy, on the other hand, describes the different rates of energy transfer to unresolvable scales resulting from each velocity component and filter direction. The former is primarily due to the largest scales of turbulence while the latter depends on the smallest resolved scales. These effects are separated in the model-split hybridization approach, and thus, separate anisotropy models can also be used.

Since stress anisotropies are mostly carried by the largest of turbulent structures, it is reasonable to expect that even small resolved turbulent kinetic energy will result in adequate total stress anisotropy in the model-splitting approach. This is, in fact, one of the main reason for hybrid modeling. An important reason for poor RANS performance is poor representation of Reynolds stress anisotropy [35]. If the hybrid approach is correctly formulated, such anisotropies should be captured in regions where sufficient resolution is provided. In this case, scalar $v_{t}$ models should be sufficient for $\tau_{i j}^{S G R S .}$. Thus, in this initial work, a standard $\overline{v^{2}}$ - $f$-based RANS model, specifically the code-friendly version[36], is used, but any of the standard models could be used. Anisotropic models for $\tau_{i j}^{S G R S}$ will be explored at a later time.

As the dissipation anisotropy is responsible for different rates of resolved energy removal in each direction, it does directly affect the structure of the resolved turbulence near the cutoff. Simulations of forced, homogeneous, isotropic turbulence with anisotropic meshes have shown that the following eddy viscosity tensor, labeled the "M43" model, gives reasonable results [21]

$$
v_{i j}^{t}=C(\mathcal{M})\langle\varepsilon\rangle^{1 / 3} \mathcal{M}_{i j}^{4 / 3},
$$

where $\langle\varepsilon\rangle$ was set equal to the power input by low-wavenumber stirring and the "constant" is a function of the eigenvalues of $\mathcal{M}$ found by fitting to the expected dissipation using the expected grad-grad product, $\left\langle\partial_{k} u_{i}^{>} \partial_{l} u_{j}^{>}\right\rangle$, assuming a $k^{-5 / 3}$ inertial range spectrum. Note that the $C(\mathcal{M})$ used here corresponds to second-order finite volume numerics and is not the same is of that presented in [34] where a psuedo-spectral code was used. The specific coefficients used here are given in the appendix. When applied to varying AR cells and shapes, (19) was shown to be greatly superior to Smagorinsky and nearly equivalent to AMD [22] (Fig. 5). This is particularly interesting because both of these previous scalar eddy viscosity forms are expressed in terms of instantaneous fluctuating velocity gradients, and therefore vary greatly and may contribute to the mean stress as there may be non-vanishing correlations. The tensor eddy viscosity (19) however, relies on no gradient information and does not fluctuate. Therefore, the fluctuating gradient portion does not contribution to the mean stress. With the $\overline{v^{2}}-f$ model used here, the required expected dissipation is set to the RANS model $\varepsilon$.

\section{Demonstration}

To demonstrate the potential of the proposed method, fully-developed, incompressible, turbulent channel flow at $R e_{\tau} \approx 5200$ is simulated. DNS data is available for this case [37] allowing evaluation of the hybrid results. A branch 


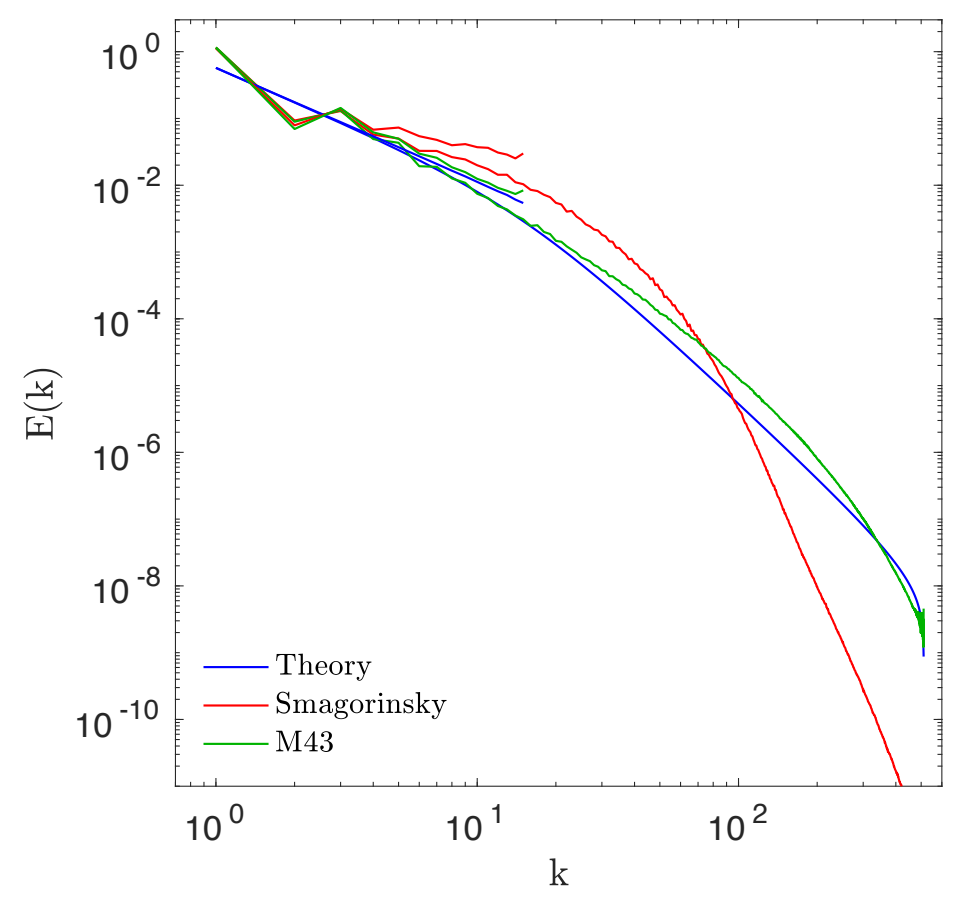

Fig. 5 Fine and coarse directional energy spectra for $(1: 32: 32)$ AR grids and infinite $R e$ forced HIT using standard Smagorinsky and (19).

of the existing finite volume incompressible Navier Stokes solver CDP v2.4 [38, 39] developed at the Stanford CTR is used here. The solver is $2^{\text {nd }}$ order in time and space with no upwinding used for convective fluxes in the momentum equation and time advancement performed with Crank-Nicolson. For the model-split hybridization, the SGRS model is based on Durbin's $\overline{v^{2}}-f$ RANS model [27] with the redistribution rate model modified as in the appendix. The SGET model used is the aforementioned M43 model 19 described in \$III.C.

The domain for the hybrid simulation is $2 \pi \times \pi$ (streamwise $\times$ spanwise), which is a substantial truncation relative to the DNS domain, which is $8 \pi \times 3 \pi$. Relative to the DNS resolution, the resolution of the hybrid simulation is reduced by a factor of $(25,10,64)$ in the streamwise, wall-normal, and spanwise directions resulting in grid spacing of $\Delta_{x}^{+} \approx 350, \Delta_{z}^{+} \approx 400$, and $\Delta_{y}^{+} \approx 300$ in the channel center with $\Delta_{y}^{+} \approx 1$ at the first cell. This combination of the domain truncation and grid coarsening leads to a grid size reduction of nearly a factor of $2 \times 10^{5}$.

For the first case, the hybrid simulation is initialized from a steady-state RANS solution. At $t_{f}=0$, the forcing is activated, and the state evolves in time from the RANS initial condition to a statistically stationary hybrid state. This evolution is summarized in Figures 6 and 7

During the first step of forcing (Fig 6, $t_{f}=0.0$ ) the resolved field is simply the RANS state and a large core of excess resolution is present as indicated by $\left\langle r_{\mathcal{M}}\right\rangle<1$. Without any resolved turbulence, $F_{i}$ is able to aggressively generate fluctuations. Note that the vector field scaling bar lengthens as time progresses indicating equivalent plotted vector lengths are decreasing in magnitude. A short time later $\left(\mathrm{Fig} 6, t_{f}=0.4\right)$, fluctuations of varying scales are present, and the forcing is reduced in response to the existing fluctuations limiting where energy can be added provided the prescribed TG structure. This trend continues through each successive snapshot while a front of $\left\langle r_{\mathcal{M}}\right\rangle \approx 1$ expands away from the wall indicating a growing region of grid-level LES. Finally, after multiple flow-throughs (Fig 6 , $t_{f}=4.0$ ), a wide spectrum of resolved fluctuations is clearly present with grid-level LES having reached the center of the channel. Throughout the simulation, $\left\langle r_{\mathcal{M}}\right\rangle$ remains large very near the wall thereby maintaining locally pure RANS mode, as expected.

Large scale structures develop more slowly (compare $\overline{u_{x}}$ at $t_{f}=0.4$ and $t_{f}=3.2$, for instance). The general idea of the proposed active forcing approach is to force the largest turbulent structures initially and gradually reduce the size of the forcing structure towards the grid length scale as those scales are populated with resolved turbulence. The 

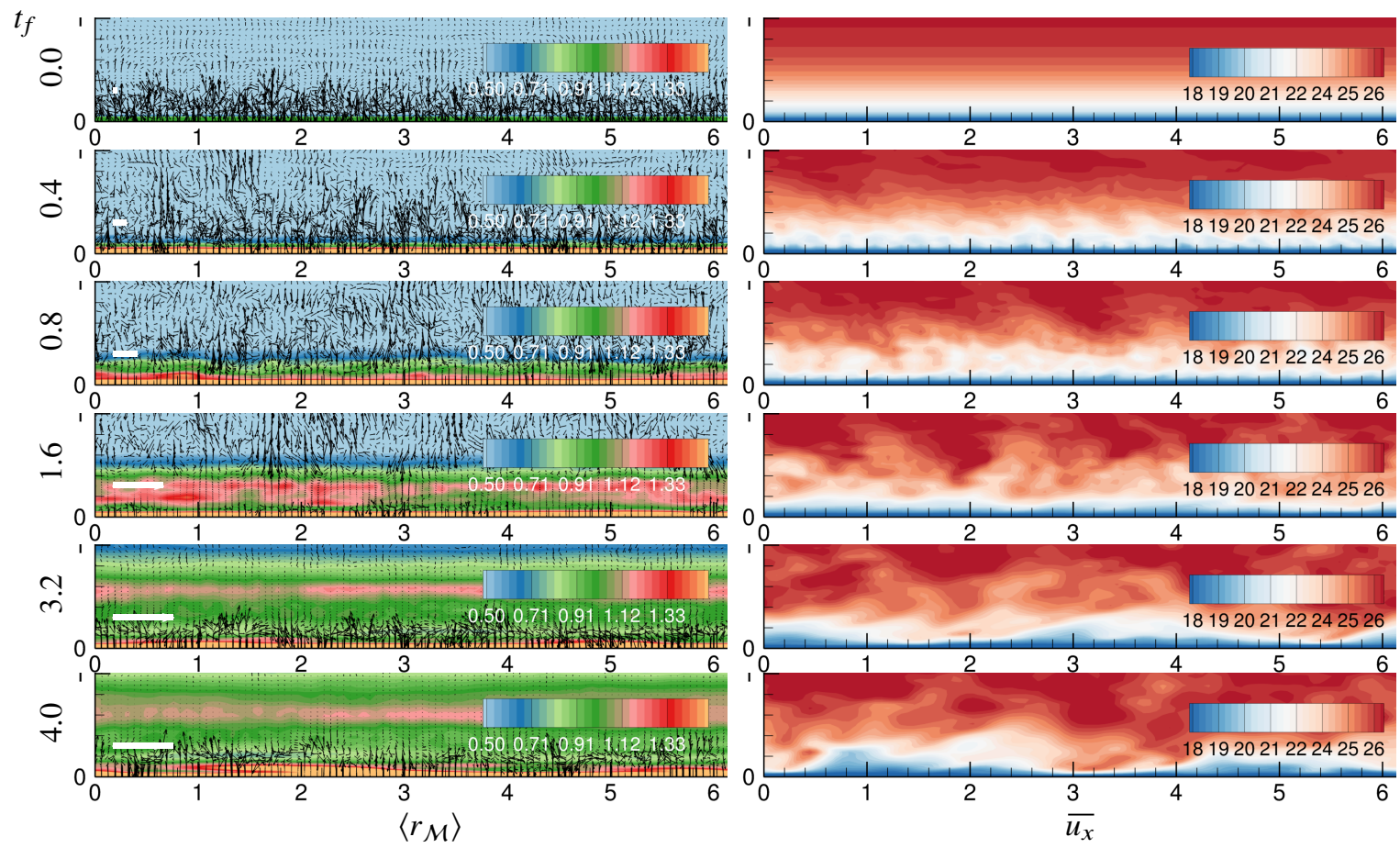

Fig. 6 Snapshots of mean resolution adequacy parameter $\left\langle r_{\mathcal{M}}\right\rangle(3)$ and total streamwise velocity for the proposed hybrid method applied to fully developed channel flow at $R e_{\tau} \approx 5200$ with forcing vector field, $F_{i}$, overlaid. Time increments are labeled with mean velocity flow throughs. Vector field scale indicated by each respective white marker bar of constant magnitude $\left(k_{\max }\right)^{1 / 2} / T_{\max }$ where the "max" subscript indicates the maiximum value over the entire domain. Half-channel shown here.

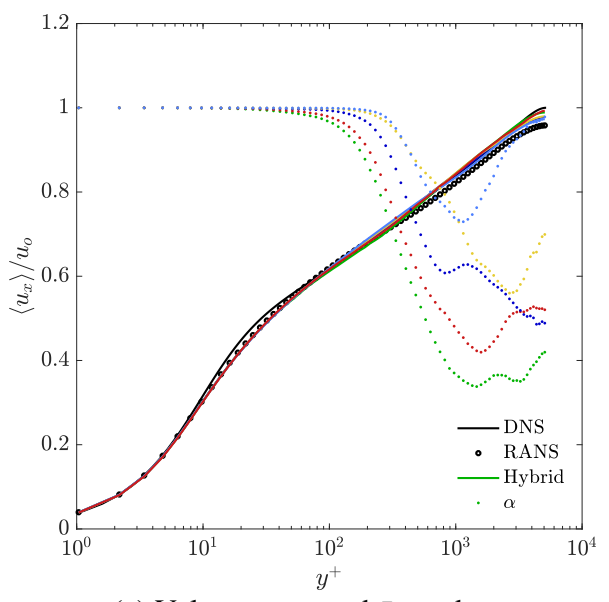

(a) Volume averaged $\bar{u}_{x}$ and $\alpha$

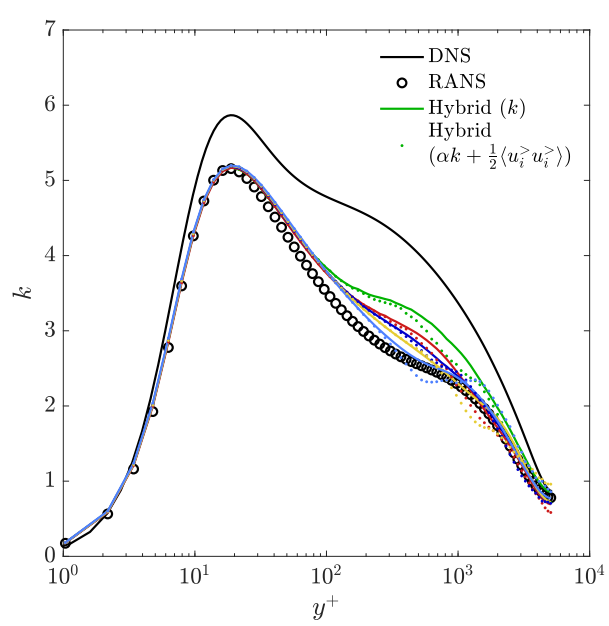

(b) Turbulent kinetic energy

Fig. 7 Normalized mean streamwise velocity for fully developed channel flow at $R e_{\tau} \approx 5200$ with overlaid level of resolved turbulence (a) and turbulent kinetic energy at the steady-state time (b). Volume averaged at times: $-0.8,-2.4,-4.0,-8.0$, and -40 flow-throughs. 
apparent deviation from the anticipated behavior indicates the scale of the TG should be increased initially, perhaps significantly.

Throughout the process of turbulence generation, the mean streamwise velocity profile remains accurate (Fig. 7a). The expected logarithmic profile is observed at all stages of development with no major deviations from the DNS data. This result is quite remarkable as for varying levels of resolved turbulence, most of which are not a grid-resolved LES, there is no apparent MSD or log-layer mismatch. Hence, the gradual body forcing approach, despite the crude form used here, appears to be sufficient for generating fluctuations that yield realistic behavior.

Results for the TKE are also encouraging (Fig. 7b). The RANS $\overline{v^{2}}-f$ result for $k$ significantly under-predicts the DNS result over most of the channel. Hybrid results are generally seen to move towards DNS in the region where some turbulence is resolved. The total turbulent kinetic energy from the RANS transport equation, $k_{\text {tot }}$, improves in response to a more accurate production term due to the contribution from the mean of the resolved stress. Comparing $k_{\text {tot }}$ to the TKE computed from the model and the resolved field-i.e., $k_{s g s}+k_{r e s}=\alpha k_{t o t}+\left\langle u_{i}^{>} u_{i}^{>}\right\rangle / 2$ - shows slight disagreement early in the forcing. However, as the simulation progresses, this modeled-resolved inconsistency decreases to only a small amount around $y^{+} \approx 1000$. This region corresponds to the slightly under-resolved band $\left(\left\langle r_{\mathcal{M}}\right\rangle>1\right)$ in Fig. 6 at $t_{f}=4.0$ and will be examined further in later work.

Next, we examine the spatially developing characteristics of the hybrid method by extending the length of the channel to $10 \pi$ and using a RANS inlet condition with convective outflow. Thus, this test case is more representative of typical applications. The grid spacings in plus units has been coarsened to $\Delta^{+} \approx 450$ in the span and streamwise directions. The results are summarized in Figures 8 .

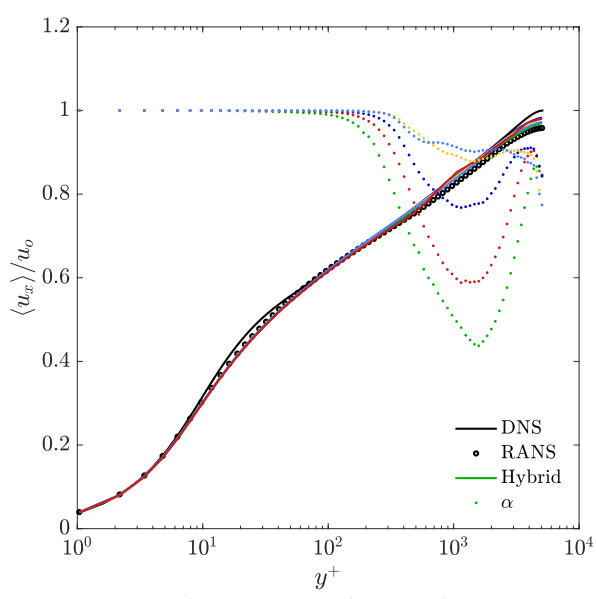

(a) Plane averaged $\bar{u}_{x}$ and $\alpha$

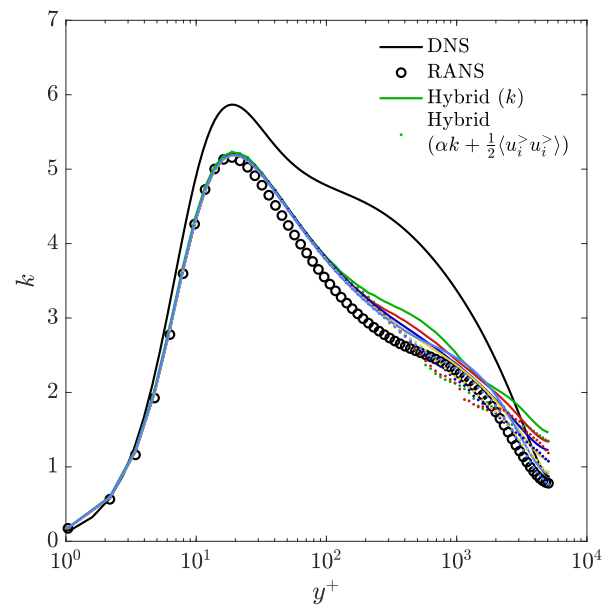

(b) Turbulent kinetic energy

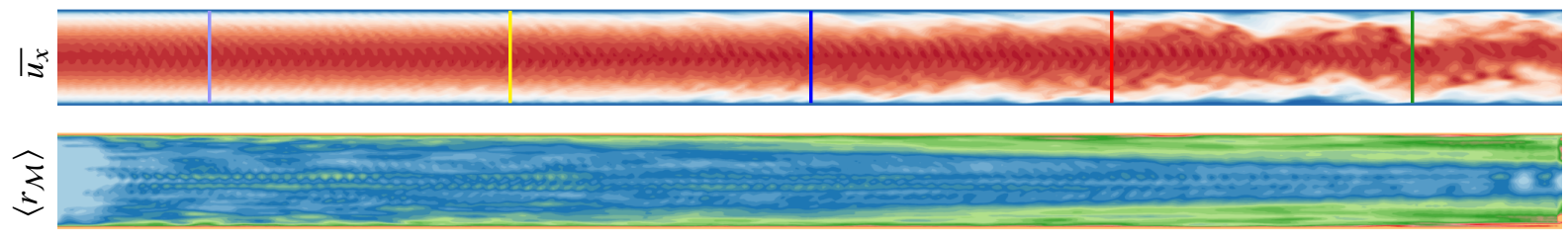

Fig. 8 Normalized mean streamwise velocity for channel flow at $R e_{\tau} \approx 5200$ with overlaid level of resolved turbulence (a) and TKE (b). Plane-averaged at positions: $-\pi,-3 \pi,-5 \pi,-7 \pi$, and $-9 \pi$ as indicated in the contour plot of $\overline{u_{x}}$. Both $\overline{u_{x}}$ and $\left\langle r_{\mathcal{M}}\right\rangle$ use the same colormap as in Fig. 6

Again, for all spatial locations and levels of resolved turbulence, the expected logarithmic profile in the mean streamwise velocity is attained (Fig. 8a). A very minor deviation is observed at the $7 \pi$ location. Resolved turbulence develops more gradually in the core of the channel in comparison to around $y^{+} \approx 1000$. This is likely due to the magnitude of the forcing scaling with the local TKE and time scale (Eq. 6). The under-utilized resolution is apparent in accompanying $\left\langle r_{\mathcal{M}}\right\rangle$ contour plot where the grid-resolved LES "front" has not extended to the channel center by the outlet. Naturally, it is highly desirable for the model to transition from RANS to grid-resolved LES with as little time 
and space as possible. Therefore, improving slow-core development is another avenue for improving future forcing formulations. Contrary to results in the temporally developing case (Fig. 7b), the TKE from both the RANS model and accounting for the contribution from the resolved, field exceeds the DNS value in the center of the channel (Fig. 8b. The reason for this inconsistency is not apparent though it does not seem to corrupt the mean flow.

These preliminary results indicate that the model-split hybridization with active forcing and SGET anisotropy is promising and worthy of further study and development.

\section{Conclusions}

By examining the failures of recently developed models, three general deficiencies of existing hybrid methods have been identified: use of a single eddy viscosity to represent both subgrid stress and energy transfer, the use of RANS-based transport models with fluctuating quantities, and the reliance on passive self-generation of resolved turbulence in hybrid regions. To remedy these, a new modeling framework is proposed. The novel aspects of the framework include 1) the model-split hybridization approach, 2) a forcing formulation which actively injects fluctuations in regions that are capable of supporting more turbulence, and 3) the ability to naturally incorporate anisotropic models, like the M43 SGET model. Preliminary results for channel flow indicate that the model leads to good predictions of the mean velocity profile for a wide range of resolutions and levels of resolved turbulence including regions of transitional, non-equilibrium developing LES. While issues related to the slow production of resolved turbulence in the channel core and slight DNS-hybrid TKE core mismatch in the spatially developing case have been identified, the approach does seem to be a solution for the common hybrid issues of log-layer mismatch and MSD.

With initial results being very encouraging, significant evaluations of more complex flow scenarios are warranted. These include common aerodynamic test cases with smooth wall separation and reattachment. Further, unsteady flows where the current time averaging procedure may prove problematic are of particular interest. In addition, many avenues for potential refinement and improvement of the TAMS approach are apparent. For example, the prescribed forcing structure may be enhanced to more closely mimic real turbulence to allow more rapid transfer of energy from modeled to resolved scales; stress anisotropy may be included to improve model performance in regions of only small amounts of resolved turbulence; the effects of turbulence anisotropy, instead of only resolution anisotropy, may be added with more advanced SGET models; and the combination of various RANS and SGET models may be exploited to leverage their individual strengths. Though early in its development, the proposed TAMS framework is a very promising approach which seems capable of achieving the hybrid RANS/LES goals of a robust, predictive, and cost-effective method of simulating complex fluid flows.

\section{Previous Models}

\section{Appendix}

Details of previous models whose failures were used to guide the requirements for the current TAMS approach are briefly detailed here. Models used to show the two opposite failure modes in the WMH (Fig. 3) retain the "Model A" and "Model B" distinctions used in the figure while the model used to illustrate the effects of including fluctuating terms in RANS equations (Fig. 1), is labeled "Model C". All three relied on passive self-generation of resolved turbulence.

Model A: This model was the earliest version which used an anisotropic resolution indicator to guide hybridization and was loosely based on the work of Perot and Gadebusch [40]. Overall hybridization was achieved with the hybrid parameter, $\alpha$, (not the same definition as used in TAMS)

$$
\begin{gathered}
D_{t} \bar{u}_{i}=-\frac{1}{\rho} \partial_{i} \bar{p}+\partial_{j}\left(\alpha \tau_{i j}\right)+v \partial_{k} \partial_{k} \bar{u}_{i} \\
D_{t} k_{m}=\alpha \mathcal{P}_{k}-\varepsilon+\partial_{i}\left[\left(v+\frac{v_{t}}{\sigma_{k}}\right) \partial_{i} k_{m}\right] .
\end{gathered}
$$

This parameter was advanced locally as

$$
\frac{d \alpha}{d t}=\frac{1}{T_{m}}\left(r_{k}-1\right)
$$


in response the initial energy-based resolution adequacy parameter

$$
r_{k}=\frac{\frac{2}{3} C_{F} \lambda_{\max }^{Q}}{\overline{v^{2}}}
$$

where $C_{F}$ is a constant which related the structure function to the subgrid kinetic energy with $\tilde{Q}_{i j}$ being a measure of the anisotropic second-order structure function

$$
\begin{gathered}
\tilde{Q}_{i j}=\mathcal{M}_{i m} Q_{m n} \mathcal{M}_{n j}, \\
Q_{i j}=\partial_{m} \bar{u}_{k} \mathcal{M}_{m i} \partial_{n} \bar{u}_{k} \mathcal{M}_{n j},
\end{gathered}
$$

and $C_{K}$ is the Kolmogorov constant. This resolution parameter attempted to compare the modeled $k_{s g s}$ with its estimate provided the information in the resolved field and resolution. The hybrid stress made use of (18) with an anisotropic eddy viscosity as

$$
v_{i j}=v_{t} a_{i j}, \quad a_{i j}=(1-\beta) \delta_{i j}+\beta\left(\frac{3}{\tilde{Q}_{k k}}\right) \tilde{Q}_{i j}^{1 / 2}, \quad \beta=\min \left(\frac{1}{r_{k}}, 1\right) .
$$

In addition to the issue identified in $\$$ II.A with using fluctuating terms in RANS-transport models, the use of a dissipation anisotropy for the entire stress, and ambiguous RANS/LES distinction, the filter-scale structure function measure in $Q_{i j}$ was found to only be valid in the limit of isotropic resolutions and gross under-estimated $k_{s g_{s}}$. Further, the construction of $Q_{i j}$ with the entire gradient is not valid in near-RANS regions.

Model B: This model was a response to the shortcomings of Model A and treated the hybridization parameter as more of a dynamic model coefficient which modified the eddy viscosity in according to the resolution adequacy parameter. Corrections to the anisotropic structure function for anisotropic grids were constructed with inertial range theory to arrive at an estimate of the expected unresolved fluctuations

$$
\left\langle u^{<} u^{<}\right\rangle \approx \max \left(C_{s f} \frac{2}{3} \zeta_{i k} \zeta_{j l} Q_{k l}\right)
$$

with $C_{s f}=0.367$, to be used for an anisotropic grid corrected resolution adequacy parameter where the anisotropic resolution correction was found using the 2/3-law,

$$
\lambda_{(i)}^{\zeta}=C_{\zeta}\left(\lambda_{(i)}^{\hat{\mathcal{M}}}\right)^{1 / 3}\left(\lambda_{(i)}^{\hat{\mathbb{Q}}}\right)^{-1 / 2}
$$

where $\mathbb{Q}=Q \varepsilon^{-2 / 3}$ and a $(\hat{*})$ indicates normalization by the appropriate power of $\delta_{\text {norm }}=\min (\mathcal{M})$. Using Cayley-Hamilton theory, the nondimensional expected gradient-gradient product is expressed as

$$
\ln \left(\lambda_{i}^{\hat{\mathbb{Q}}}\right)=\sum_{p=0}^{2} g_{p} \ln \left(\lambda_{i}^{\hat{\mathcal{M}}}\right)^{p}
$$

where the coefficients $g_{i}$, are function of the eigenvalues of $\mathcal{M}$. Similar to the M43 model, fourth-order polynomials of the scaled eigenvalues of $\mathcal{M}$ expressed as $r=\left(a^{2}+b^{2}\right)^{1 / 2}$ and $\theta=\cos ^{-1}(\max (a, b) / r)$ with $a=\lambda_{\neq n o r m+1}^{\hat{\mathcal{M}}}$ and $b=\lambda_{\neq \text {norm }-1}^{\hat{\mathcal{M}}}$, i.e. the other two eigenvalues not $\delta_{\text {norm }}$ (though these may be repeating) and $y=\ln (\sin (2 \theta)), x=\ln (r)$ with

$$
\begin{aligned}
g_{i}= & c_{i}(1)+c_{i}(2) x+c_{i}(3) y+c_{i}(4) x^{2}+c_{i}(5) x y+c_{i}(6) y^{2}+c_{i}(7) x^{3}+c_{i}(8) x^{2} y+ \\
& c_{i}(9) x y^{2}+c_{i}(10) y^{3}+c_{i}(11) x^{4}+c_{i}(12) x^{3} y+c_{i}(13) x^{2} y^{2}+c_{i}(14) x y^{3}+c_{i}(15) y^{4}
\end{aligned}
$$

were used. Data used to fit $g$-coefficients was found from numerical integration of twice the gradient of the inertial range spectral energy density tensor integrated from $k_{\min }$ to $k_{c}$ with $k_{c}$ corresponding to a wide range of filter anisotropies and a constant ratio of the coarsest filter width to $k_{\min }$ of 32 . The coefficients in $g_{i}$ depend on the 
numerical method in use; filtering in the numerical data calculations was performed according. We find
$c_{0}(1)=-1.344$
$c_{1}(1)=1.953$
$c_{2}(1)=-0.01496$
$c_{0}(2)=-1.299$
$c_{1}(2)=0.03871$
$c_{2}(2)=0.002111$
$c_{0}(3)=0.3625$
$c_{1}(3)=-0.003524$
$c_{2}(3)=-0.005774$
$c_{0}(4)=-0.01143$
$c_{1}(4)=-0.009029$
$c_{2}(4)=0.0002191$
$c_{0}(5)=-0.0002359$
$c_{1}(5)=0.007419$
$c_{2}(5)=0.0006652$
$c_{0}(6)=0.2315$
$c_{1}(6)=0.001213$
$c_{2}(6)=-0.001771$
$c_{0}(7)=0.001695$
$c_{1}(7)=0.001038$
$c_{2}(7)=-0.00008604$
$c_{0}(8)=-0.0002870$
$c_{1}(8)=-0.001149$
$c_{2}(8)=0.000003282$
$c_{0}(9)=-0.0009096$
$c_{1}(9)=0.0008780$
$c_{2}(9)=0.0001955$
$c_{0}(10)=0.06177$
$c_{1}(10)=0.0006097$
$c_{2}(10)=-0.0002762$
$c_{0}(11)=-0.00009189$
$c_{1}(11)=-0.00004717$
$c_{2}(11)=0.000006200$
$c_{0}(12)=0.00005443$
$c_{1}(12)=0.00006688$
$c_{2}(12)=-0.000004179$
$c_{0}(13)=0.0001554$
$c_{1}(13)=-0.00004775$
$c_{2}(13)=-0.000009257$
$c_{0}(14)=0.0001159$
$c_{1}(14)=0.00005497$
$c_{2}(14)=0.00001253$
$c_{0}(15)=0.005991$
$c_{1}(15)=0.00007598$
$c_{2}(15)=-0.00001806$

The dynamic coefficient equation was altered to be an independent transport model for $\alpha$ as

$$
\partial_{t} \alpha+\bar{u}_{j} \partial_{j} \alpha=\frac{1}{T_{\alpha}}\left(S_{r}-D_{r}-F_{r}\right)+\frac{S_{c}}{T_{c}}+\partial_{k}\left(\frac{v_{t}}{\sigma_{\alpha}} \partial_{k} \alpha\right)
$$

where

$$
\begin{gathered}
S_{r}=\left\{\begin{array}{cc}
\tanh \left(r_{k}-1\right) & \text { if } r_{k} \geq 1, \\
\tanh \left(1-\frac{1}{r_{k}}\right) & \text { if } r_{k}<1,
\end{array}\right. \\
D_{r}=w_{\text {rans }} \max \left(\alpha+S_{r}-1,0\right), \\
w_{\text {rans }}=\tanh \left(\frac{1}{2} \max \left(r_{\varepsilon}-1,0\right)^{1 / 4}\right), \\
F_{r}=\min \left(\alpha+S_{r}, 0\right), \\
T_{\alpha}=C_{\alpha} \max \left(\frac{k}{\varepsilon}, C_{T}\left(\frac{v}{\varepsilon}\right)^{1 / 2}\right), \\
S_{c}= \begin{cases}1 & \text { if } S_{r} \& T_{c} \geq 0, \\
0 & \text { otherwise, }\end{cases} \\
T_{c}=\frac{L_{m}}{\max \left(\bar{u}_{j} \partial_{j} \mathcal{M}_{m n}\right)},
\end{gathered}
$$

and two parameters which compare the estimates of unresolved turbulent fluctuations and turbulent dissipation are respectively

$$
r_{k}=C_{k Q} C_{s f} \frac{4}{9} \frac{k}{\overline{v^{2}}} \frac{\max \left(\zeta_{i m} Q_{m n} \zeta_{n j}\right)}{\overline{v^{2}}}
$$

and

$$
r_{\varepsilon}=C_{\varepsilon} r_{k}^{3 / 2} \frac{L_{m}}{\delta_{\max }}
$$

where $\delta_{\max }$ is the magnitude of the projection of $\mathcal{M}$ on the direction of the maximum eigenvalue of $\zeta_{i m} Q_{m n} \zeta_{n j}$. Coefficients were empirical tuned as $\sigma_{\alpha}=10, C_{\alpha}=4, C_{k Q}=8$, and $C_{\varepsilon}=0.03125$. Initial condition are set to basic RANS with $\alpha_{o}=1$ as well as wall and inlet boundary conditions $\left.\alpha\right|_{\text {wall }}=1,\left.\alpha\right|_{\text {inlet }}=1$, while outlet conditions are 
set to standard convective outflow, $\left.\partial_{n} \alpha\right|_{\text {outflow }}=0$. Anisotropy in $v_{i j}$ was modified as

$$
a_{i j}=w_{R A N S} \delta_{i j}+\left(1-w_{R A N S}\right) \sqrt{3}|Q|^{-1} Q_{i j}
$$

which was again scaled by $v_{t}$ and $\alpha$ to arrive at $\tau_{i j}$. Model B still suffered from all the main shortcomings identified in Model A with the exception of correcting the tensor structure function for anisotropic resolution. Though, it is worth pointing out that the ad-hoc RANS/LES blending in (33) and (40), when coupled with the anisotropy in $Q_{i j}$ resulted in the nearly correct $C_{f}$ leading to the top of the hump (Fig. 44). This $C_{f}$ feature has not been captured in other simulations (e.g. [41]). While it was no appropriate to the use grid-based length to formulate a RANS-region stress anisotropy, the grid changing lengthscale near the wall may have been mimicking the change in the turbulent lengthscale. These results suggest anisotropy based on contraction of the resolved gradient-gradient product, with the correct turbulent lengthscale, may lead to improved basic RANS.

Model C: The primary modification in Model C was a strict reinterpretation of the hybridization parameter as the ratio of modeled to total TKE, or $\alpha=k_{s g s} / k_{t o t}$, as in other models such as PANS [6]. Starting from a Deardorff $k_{s g s}$ transport equation,

$$
D_{t} k_{s g s}=\alpha^{2} \mathcal{P}_{s g s}-\varepsilon+\partial_{i}\left(\alpha^{2} v_{t} \partial_{i} k_{s g s}\right),
$$

where $v_{t}=C_{\mu} k_{t o t}^{2} / \varepsilon, \mathcal{P}_{s g s}=2 \alpha^{2} v_{t} \bar{S}_{i j} \bar{S}_{i j}$, the supposedly equivalent $k_{t o t}$ equation was derived with $D_{t} k_{s g s}=$ $D_{t}\left(\alpha k_{t o t}\right)$ as

$$
D_{t} k_{t o t}=\alpha \mathcal{P}_{s g s}-\frac{k_{t o t}}{\alpha} D_{t} \alpha-\frac{\varepsilon}{\alpha}+\frac{1}{\alpha} \partial_{i}\left(v_{t} \partial_{i}\left(\alpha k_{t o t}\right)\right)
$$

The evolution of the level of resolved turbulence was modeled as in Model B (30). Further, the resolution adequacy parameter was changed to the legthscale-based approach as in (3). Again, this method failed to recognize the deleterious effects of including fluctuating quantities in non-linear RANS source terms as well as using the standard merging mean and fluctuating contributions to $\tau_{i j}$ with a single eddy viscosity. Further, as this model explicitly included a sink term in $k_{t o t}$ due to the total change in $\alpha$, it implicitly assumed that precise amount of resolved $k$ was being added. This is, of course, not possible when relying on passive self-generation.

\section{Current model details}

For completeness, the models used in the presented results are provided here. The SGS portion of the MS formulation makes use of the "code-friendly" version [36] of Durbin's $\overline{v^{2}}-f$ model [27]. The three associated equations for turbulent kinetic energy, $k$, turbulent dissipation rate, $\varepsilon$, and minimum turbulent stress component, $\overline{v^{2}}$, take the generic form

$$
D_{t} \phi=\mathcal{P}_{\phi}-\mathcal{D}_{\phi}+\mathcal{V}_{\phi}
$$

where

$$
\mathcal{V}_{\phi}=\partial_{k}\left(\left(v+\sigma_{\phi} v_{t}\right) \partial_{k} \phi\right) .
$$

With the eddy viscosity as $v_{t}=C_{\mu} \overline{v^{2}} T$, source terms, wall boundary conditions, and diffusion coefficients are as follows

$$
\begin{aligned}
& \mathcal{P}_{k}=2 v_{t} S^{2} \\
& \mathcal{P}_{\varepsilon}=\frac{\mathcal{P}_{k}}{T} \\
& \mathcal{D}_{k}=\varepsilon \\
& \sigma_{k}=1 \\
& k_{\text {wall }}=0 \\
& \mathcal{D}_{\varepsilon}=\frac{\varepsilon}{T} \\
& \mathcal{P}_{v 2}=k f \\
& \sigma_{\varepsilon}=1 / 1.3 \\
& \varepsilon_{\text {wall }}=2 v \frac{k^{2}}{\delta_{\text {wall }}^{2}} \\
& \begin{aligned}
\mathcal{D}_{v 2} & =6 \frac{\overline{v^{2}}}{k} \varepsilon \\
\sigma_{v 2} & =1 \\
\bar{v}_{\text {wall }} & =0
\end{aligned}
\end{aligned}
$$

where $S=\left(\left\langle S_{i j}\right\rangle\left\langle S_{i j}\right\rangle\right)^{1 / 2}, \delta_{\text {wall }}$ is the distance to the first cell center, and the turbulence time scale is

$$
T=\min \left(\max \left(\frac{k}{\varepsilon}, 6 \frac{\sqrt{v}}{\varepsilon}\right), \frac{0.6 k}{\sqrt{6} C_{\mu} \overline{v^{2}} S}\right) .
$$


The model for the redistribution rate, $f$, appearing in the production of $\overline{v^{2}}$ is of the Helmholtz type

$$
\left(C_{L} L\right)^{2} \partial_{k} \partial_{k} f-f=R_{f}\left(\frac{\overline{v^{2}}}{k}\left(C_{1}-6\right)-\frac{2}{3}\left(C_{1}-1\right)\right)-C_{2} \frac{\mathcal{P}_{k}}{k}
$$

where

$$
L=\max \left(\min \left(\frac{k^{3 / 2}}{\varepsilon}, \frac{k^{3 / 2}}{\sqrt{6} C_{\mu} \overline{v^{2}} S}\right), C_{\eta} \frac{v^{3 / 4}}{\varepsilon^{1 / 4}}\right)
$$

and an additional time scale modification is made here with

$$
R_{f}=\min \left(\frac{1}{T}, \frac{S}{3}\right)
$$

which was found to improve behavior for higher Re channel flow. Without this modification, $\overline{v^{2}}$ becomes excessive in hybrid simulations in response to $k$ increasing towards the DNS value while the basic RANS $\overline{v^{2}}$ is already near the DNS value. Due to how $r_{\mathcal{M}}$ is formulated based on $\overline{v^{2}}$, this error resulted in forcing being activated too near the wall. Therefore, when not using the modification in (48), additional near-wall protection is necessary. An additional scaling-factor of

$$
\eta_{\text {wall }}=\frac{1}{2}\left(\tanh \left(C_{\zeta}\left(\zeta-\zeta_{c}\right)\right)+1\right)
$$

applied to 17 , with $\zeta=1.5 \overline{v^{2}} / k$, was found to be sufficient. The model is complete with the following coefficients

$$
\begin{aligned}
C_{\mu} & =0.2, C_{1}=1.4, C_{2}=0.3, C_{L}=0.23, \\
C_{\varepsilon 1} & =1.4\left(1+0.005\left(k / \bar{v}^{2}\right)^{1 / 2}\right), C_{\varepsilon 2}=1.9, \\
C_{\eta} & =70, C_{\zeta}=10, \zeta_{c}=0.55
\end{aligned}
$$

M43 SGET: The M43 model form is provided in $(19)$. Here, $C(\mathcal{M})$ is provided. Again, using the polynomial form of 29), coefficients for $2^{\text {nd }}$-order finite volume numerics are

$$
\begin{array}{r}
c=(1.034,-0.1541,-0.007737,0.1776,0.06087,0.1622,-0.04109,-0.02738, \\
0.005521,0.04914,0.002926,0.002673,0.0004864,0.002136,0.005113)
\end{array}
$$

with $C_{\mathcal{M}}^{\circ}=0.13$. Naturally, the use of polynomials to fit the coefficients limits their applicability to the range of fitting data. The above coefficients make us of data up to $(128: 1)$ aspect ratio cells. Further, $C(\mathcal{M})$ is scaled by $\max \left(\min \left(\left\langle r_{\mathcal{M}}\right\rangle, 10\right), 1\right)$ to rapidly remove resolved fluctuations where resolved turbulence has been transported into regions with insufficient resolution. This modification is essentially ad-hoc and methods of removing it will be studied in future work.

\section{Acknowledgements}

This material is based upon work supported by NASA under cooperative agreement number NNX15AU40A.

\section{References}

[1] Spalart, P., Deck, S., Shur, M., Squires, K., Strelets, M., and Travin, A., "A new version of detached-eddy simulation, resistant to ambiguous grid densities," Theoretical Computational Fluid Dynamics, Vol. 20, 2006, pp. 181-195.

[2] Shur, M., Spalart, P., Strelets, M., and Travin, A., "A Hybrid RANS-LES approach with delayed-DES and wall-modelled LES capabilities," International Journal of Heat and Fluid Flow, Vol. 29, 2008, pp. 1638-1649.

[3] Riou, J., Garnier, E., Deck, S., and Basdevant, C., "An extension of DDES applied to the computation of a generic missle fin 
in transonic regime," Proceedings of the 7th International Symposium on Engineering Turbulence Modeling in Measurements, 2008, pp. 732-737.

[4] Gritskevich, M., Garbaruk, A., Schutze, J., and Menter, F., "Development of DDES and IDDES formulations for the $k-\omega$ shear stress transport model," Flow Turbulence Combustion, Vol. 88, 2012, pp. 431-449.

[5] Jee, S., and Shariff, K., "Detached-eddy simulation based on the $v^{2}-f$ model," Seventh International Conference on Computational Fluid Dynamics, 2012.

[6] Girimaji, S. S., "Partially-Averaged Navier-Stokes Model for Turbulence: A Reynolds-Averaged Navier-Stokes to Direct Numerical Simulation Bridging Method," Journal of Applied Mechanics, Vol. 73, 2005, pp. 413-421.

[7] Foroutan, H., and Yavuzkurt, S., "A partially averaged Navier Stokes model for the simulation of turbulent swirling flow with vortex breakdown," Int. J. Heat Fluid Flow, Vol. 50, 2014, pp. 402-416.

[8] Chaouat, B., and Schiestel, R., "A new partially integrated transport model for subgrid-scale stresses and dissipation rate for turbulent developing flows," Physics of Fluids, Vol. 17, 2005, p. 065106.

[9] Chaouat, B., "The State of the Art of Hybrid RANS/LES Modeling for the Simulation of Turbulent Flows," Flow Turbulence Combust, Vol. 99, 2017, pp. 279-327.

[10] Menter, F. R., and Egorov, Y., “A Scale Adaptive Simulation Model Using Two-Equation Models,” AIAA Paper 2005-1095, 2005.

[11] Menter, F. R., and Egorov, Y., "The Scale-Adaptive Simulation Method for Unsteady Turbulent Flow Predictions. Part 1: Theory and Model Description," Flow, Turbulence and Combustion, Vol. 85, No. 1, 2010, pp. 113-138. doi: 10.1007/s10494-010-9264-5, URL https://doi .org/10.1007/s10494-010-9264-5.

[12] Quemere, P., and Sagaut, P., “Zonal multi-domain RANS/LES simulations of turbulent fows," Int. J. Numerical Methods in Fluids, Vol. 40, 2002, pp. 903-925.

[13] Deck, S., "Zonal-Detached-Eddy Simulation of the Flow Around a High-Lift Configuration," AIAA Journal, Vol. 43, No. 11, 2005.

[14] Frohlich, J., and von Terzi, D., "Hybrid LES/RANS methods for the simulation of turbulent flows," Progress in Aerospace Sciences, Vol. 44, 2008, pp. 349-377.

[15] Bhushan, S., and Walters, D. K., "A dynamic hybrid Reynolds-averaged Navier Stokes-Large eddy simulation modeling framework," Physics of Fluids, Vol. 24, 2012.

[16] Xiao, H., Wang, J.-X., and Jenny, P., "An Implicitly Consistent Formulation of a Dual-Mesh Hybrid LES/RANS Method," Commun. Comput. Phys., Vol. 21, No. 2, 2017, pp. 570-599.

[17] Spalart, P., "Detached-eddy simulation,” Annual Review of Fluid Mechanics, Vol. 41, 2009, pp. 181-202.

[18] Deardorff, J. W., "The use of subgrid transport equations in a three-dimensional model of atmospheric turbulence," Journal of Fluids Engineering, 1973.

[19] Jimenéz, J., and Moser, R. D., "Large Eddy Simulation: Where are we and what can we expect?" AIAA Journal, Vol. 38, No. 4, 2000, pp. 605-612.

[20] Greenblatt, D., Paschal, K. B., Yao, C., Harris, J., Schaeffler, N. W., and Washburn, A., "Experimental Investigation of Separation Control Part 1: Baseline and Steady Suction,” AIAA Journal, Vol. 44, No. 12, 2006, pp. 2820-2830.

[21] Haering, S., and Moser, R. D., "Resolution-induced anisotropy in LES," In preparation, 2018.

[22] Rozema, W., Bae, H. J., Moin, P., and Verstappen, R., "Minimum-dissipation models for large-eddy simulation,” Physics of Fluids, Vol. 27, 2015, p. 085107.

[23] Lumley, J. L., "Similarity and turbulent energy spectrum,” The Physics of Fluids, Vol. 10, No. 4, 1967.

[24] Lesieur, M., and Metais, O., "New trends in large-eddy simulations of turbulence," Annual Review of Fluid Mechanics, Vol. 28, 1996, pp. 245-82.

[25] Spalart, P., and Allmaras, A., "A one-equation turbulence model for aerodynamic flows," La Recherche Aerospatiale, Vol. 1, 1994, pp. 5-21. 
[26] Synge, J. L., and Schild, A., Tensor Calculus, Dover, 1949.

[27] Durbin, P., "Separated flow computations with the $k-\varepsilon-v^{2}$ model," AIAA Journal, Vol. 33, No. 4, 1995, pp. $659-664$.

[28] Shur, M. L., Spalart, P. R., Strelets, M. K., and Travin, A. K., "Synthetic Turbulence Generators for RANS-LES Interfaces in Zonal Simulations of Aerodynamic and Aeroacoustic Problems," Flow, Turbulence and Combustion, Vol. 93, 2014, pp. 63-92. doi:10.1007/s10494-014-9534-8, URL https://doi.org/10.1007/s10494-014-9534-8.

[29] Spalart, P. R., Belyaev, K. V., Garbaruk, A. V., Shur, M. L., Strelets, M. K., and Travin, A. K., "Large-eddy and Direct Numerical Simulations of the Bachalo-Johnson flow with shock-induced separation," Flow, Turbulence and Combustion, Vol. 99, 2017, pp. 865-885.

[30] Menter, F., Garbaruk, A., Smirnov, P., Cokljat, D., and Mathey, F., "Scale-adaptive simulation with artificial forcing," Progress in Hybrid RANS-LES Modelling, 2010, pp. 235-246.

[31] Kraichnan, R. H., "Diffusion by a random velocity field," Physics of Fluids, Vol. 13, No. 1, 1969, pp. $22-31$.

[32] Batten, P., Goldberg, U., and Chakravarty, S., "Interfacing statistical turbulence closures with large-eddy simulation," AIAA Journal, Vol. 42, 2004, pp. 485-492.

[33] Haering, S. W., "Anisotropic Hybrid Turbulence Modeling with Specific Application to the Simulation of Pulse-Actuated Dynamic Stall Control," Ph.D. thesis, The University of Texas at Austin, 2015.

[34] Moser, R., "Novel Hybrid RANS/LES Models for Aerodynamic Flows: Year 2 Technical Report," Tech. rep., NASA Cooperative agreement number: NNX15AU40A, 2017.

[35] Launder, B. E., and Rodi, G. J. R. W., "Progress in the development of a Reynolds-stress turbulent closure," Journal of Fluid Mechanics, Vol. 68, No. 3, 1975, pp. 537-566.

[36] Lien, F., and Kalitzin, G., "Computations of transonic flow with the $v^{2}-f$ turbulence model," Heat and Fluid Flow, Vol. 22, 2001, pp. 53-61.

[37] Lee, M. K., and Moser, R. D., "Direct numerical simulation of turbulent channel flow up to $\operatorname{Re}_{\tau} \approx 5200$," Journal of Fluid Mechanics, Vol. 774, 2015, pp. 395-415.

[38] Ham, F., and Iaccarino, G., "Energy conservation in collocated discretization schemes on unstructured meshes," Center for Turbulence Research Annual Research Briefs, 2004.

[39] You, D., Ham, F., and Moin, P., "Discrete conservation principles in large-eddy simulation with application to separation control over an airfoil," Physics of Fluids, Vol. 20, 2008.

[40] Perot, J. B., and Gadebusch, J., "A self-adapting turbulence model for flow simulation at any mesh resolution," Physics of Fluids, Vol. 19, 2007.

[41] You, S. D., Wang, M., and Moin, P., "Large-Eddy Simulation of Flow over a Wall-Mounted Hump with Separation Control," AIAA Journal, Vol. 44, No. 11, 2006, pp. 2571-2577. 